\title{
Minerva: User-Centered Science Operations Software Capability for Future Human Exploration
}

\author{
Matthew Deans \\ NASA Ames Research \\ Center \\ Moffett Field, CA 94035 \\ matthew.deans@nasa.gov \\ Ivonne Deliz \\ ASRC / NASA Ames \\ Research Center \\ Moffett Field, CA 94035 \\ ivy.deliz@nasa.gov
}

\author{
Jessica J. Marquez \\ NASA Ames Research \\ Center \\ Moffett Field, CA 94035
}

jessica.j.marquez@nasa.gov

Steven Hillenius
NASA Ames Research
Center
Moffett Field, CA 94035
steven.r.hillenius@nasa.gov

\author{
Tamar Cohen \\ SGT Inc. / NASA Ames \\ Research Center \\ Moffett Field, CA 94035 \\ tamar.e.cohen@nasa.gov \\ Jeffrey Hoffman \\ Massachusetts Institute of \\ Technology \\ Cambridge, MA 02139 \\ jhoffma1@mit.edu
}

\author{
Matthew J. Miller \\ Georgia Institute of \\ Technology \\ Atlanta, GA 30332 \\ mmiller@gatech.edu \\ Yeon Jin Lee \\ SG Inc. / NASA Ames \\ Research Center \\ Moffett Field, CA 94035 \\ yeonjin.lee@nasa.gov
}

\author{
David Lees \\ Carnegie Mellon Silicon \\ Valley \\ Moffett Field, CA 94035 \\ david.s.lees@nasa.gov
}

\author{
Johannes Norheim \\ Massachusetts Institute of \\ Technology \\ Cambridge, MA 02139 \\ norheim@mit.edu
}

\author{
Darlene S. S. Lim \\ BAERI / NASA Ames \\ Research Center \\ Moffett Field, CA 94035 \\ darlene.lim@nasa.gov
}

\begin{abstract}
In June of 2016, the Biologic Analog Science Associated with Lava Terrains (BASALT) research project conducted its first field deployment, which we call BASALT-1. BASALT-1 consisted of a science-driven field campaign in a volcanic field in Idaho as a simulated human mission to Mars. Scientists and mission operators were provided a suite of ground software tools that we refer to collectively as Minerva to carry out their work. Minerva provides capabilities for traverse planning and route optimization, timeline generation and display, procedure management, execution monitoring, data archiving, visualization, and search. This paper describes the Minerva architecture, constituent components, use cases, and some preliminary findings from the BASALT-1 campaign.
\end{abstract}

\section{TABle of Contents}

1. INTRODUCTION ............................................................ 1

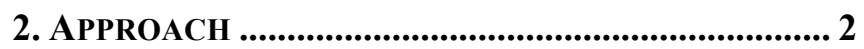

3. FIELD TESTING ....................................................... 5

4. RESULTS ..................................................................... 8

5. FUTURE WORK ................................................... 10

6. ACKNOWLEDGEMENTS .......................................... 10

REFERENCES...................................................... 10

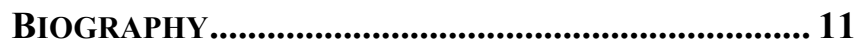

\section{INTRODUCTION}

NASA's flexible architecture for human exploration is exemplified by various Design Reference Mission (DRM) studies, which outline plans for the exploration of various targets including lunar and cis-lunar environments, Near Earth Asteroids (NEAs), Phobos \& Deimos, and Mars [1-3]. Surface science and exploration with humans and robotic assets are ubiquitous elements of each of these mission scenarios. One challenge for each of these prospective

U.S. Government work not protected by U.S. copyright. destinations is: How can scientific return be maximized given the operational constraints associated with deep space exploration? With crew time and resources at a premium, it is critically important to develop, test and validate science support capabilities and operational concepts to optimize human architecture planning and science systems including instruments, communications, ground control, and personnel organization.

Terrestrial analog campaigns provide a contextually relevant environment in which to test and validate capabilities that address these architectural issues. Science-driven analogs, such as the BASALT (Biologic Analog Science Associated with Lava Terrains) research project, are critically important to this process. ${ }^{1}$ The BASALT science program is focused on understanding habitability conditions of early and present-day Mars in two relevant Mars analog locations (the East Rift Zone (ERZ) flow on the Big Island of Hawai'i and the eastern Snake River Plain (ESRP) in Idaho). The objective is to characterize and compare the physical and geochemical conditions of life in these analog environments and to learn how to seek, identify, and characterize life and life-related chemistry in basaltic environments representing two epochs of Martian history.

Scientific fieldwork is conducted under simulated Mars mission constraints, including simulated extravehicular activity (EVA) to evaluate selected conops (concepts of operations) and capabilities with respect to their anticipated value for the joint human and robotic exploration of Mars. By conops we mean operational design elements that guide the organization and flow of hardware, personnel, communications, and data products through the course of a mission. By capabilities we mean functionalities that can take the form of hardware or software. For a complete description of BASALT-1 ConOps, see [15]. Given that our

\footnotetext{
${ }^{1}$ See: https://spacescience.arc.nasa.gov/basalt/ for more BASALT details
} 
scientific goals are not simulated, there is intrinsic pressure on each field deployment to maximize scientific return. This requires thoughtful and directed mission planning and scheduling, traverse path planning, physical sample collection, sample curating, and data assimilation and dissemination. The management of these mission elements affects both tactical and strategic aspects of our field deployments. In addition to our BASALT experience, previous analog projects such as the Pavilion Lake Research Project (PLRP) [4,5], NASA Extreme Environment Mission Operations (NEEMO) [6-8], and Mojave Volatiles Prospector (MVP) [9] have further demonstrated the need for high-performance capabilities that meet these scientific operational mission requirements.

\section{APPROACH}

There are a number of separate capabilities in the community for traverse planning, crew scheduling, optimization, navigation, communication, decision-making, sample documentation, and science team utilization in service of scientific mission management requirements [1013]. To date there has not been an integrated suite of complementary science operations systems that will provide all of these capabilities in a seamless and user-centered manner. A key objective of the BASALT project is to design, test and deploy such an integrated system while evolving its support capabilities to meet human and robotic mission requirements specific to deep space and Mars scientific exploration. Our integrated capability is referred to collectively as Minerva (after the Roman goddess of wisdom).

Minerva brings together existing pieces of software by integrating workflows and providing interfaces between preexisting tools. Our goal is to make the Minerva suite of tools usable and effective, particularly in service of managing science operations and enabling science return. These constituent components include Playbook, xGDS (Exploration Ground Data Systems), and SEXTANT.

\section{Playbook}

Playbook is a mobile schedule viewer and real-time execution software aid [13] which has been used at a diverse set of analog missions: PLRP, NEEMO, MVP, Desert Research and Technologies Studies (D-RATS), Deep Space Habitat (DSH), Space Environment Analog for Testing EVA Systems and Training (SEATEST), Regolith and Environment Science and Oxygen and Lunar Volatile Extraction (RESOLVE), and Human Exploration Research Analog (HERA). Over three years, we have incrementally developed Playbook to better support the various needs of Earth analog missions. Over the course of the BASALT project, we plan to evaluate existing capabilities and subsequently add new capabilities to enable integrated science and astronaut teams to conduct Mars-like EVA. Playbook also incorporates data transmission delay between ground teams and analog Mars Crew team.

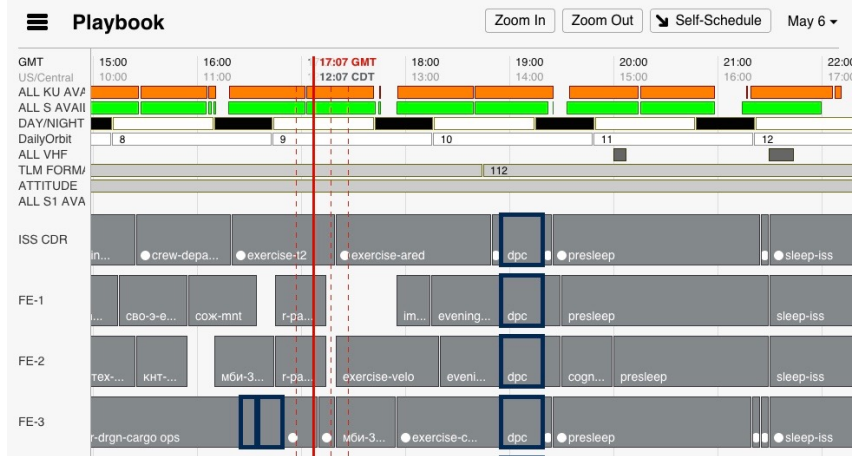

Figure 1. Playbook timeline, red lines track times

Playbook has three main functions available to BASALT: Timeline viewing (Figure 1), Mission Log text communication interface (Figure 2), and the Procedure repository. The Procedure repository provides online access to procedures, flight rules, and EVA priority documentation. The Timeline view shows the scheduled times for all the critical mission activities as well as the EVA timeline phases for each role: Intravehicular Crew (IV), Extravehicular Crew (EV), and Science Team (ST). This collaborative view allows the EV/IV crew, Mission Support Center (MSC), including ST and other support personnel, to share the same dynamic timeline. The Timeline view provides aids to help understand the communication latency between teams: a solid red line represents current time and three dashed red lines represent current time minus one-way latency time, current time plus one-way latency time, and current time plus round trip latency time.

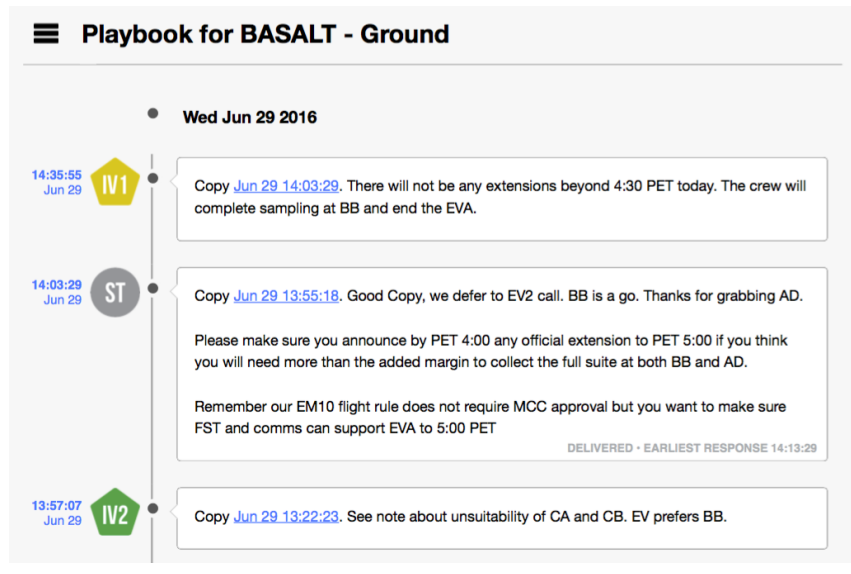

Figure 2. Playbook Mission Log with messages

The Mission Log is a chat interface where the MSC and IV teams can share text, files, images, and video messages. Leveraged significantly when data transmissions are delayed, it has become a key capability for teams to communicate during the EVA. Within the MSC, the ST Lead was tasked with posting messages to the IV crew through the Mission Log. These transmissions were carefully scripted to efficiently represent the consensusbased decisions and guidelines that were to be conveyed to the EV/IV crew. Messages included directives regarding sampling priorities, scientific queries and requests for 
clarification, among other critical information sharing that was focused on improving the scientific quality of the EVAs. The IV crew were also able to add further descriptive and operational details about the EVA not necessarily discernible to the MSC from transmitted voice, video, and still images. Each message is time-stamped and has a countdown timer to show the sender when to expect their message to arrive and the earliest time to receive a response.

Playbook typically runs on a cloud based architecture and uses a completely web based interface. This allows rapid deployment for mission analogs where quickly installing or setting up software for many users can be challenging. Users only need a modern web browser to access Playbook for a given mission. For the BASALT analogs, because of the remote nature and limited internet connectivity, Playbook was deployed on a physical server on site for the mission. Because Playbook is primarily client side software which relies on a small efficient server process, we are able to deploy and support a large number of users $(\sim 50+)$ on modest commodity hardware, such as a laptop acting as a server.

\section{$x G D S$}

Exploration Ground Data Systems (xGDS) is a set of opensource, web-based tools including mapping capabilities for sharing, authoring, and visualizing map layers; a traverse plan editor for planning field activities in a geospatial context; displays for real-time visualization of vehicle and crew location and activity, system telemetry, and payload data; and an integrated data archive for post-hoc analysis, browsing, and search. xGDS capabilities have been matured by deployment at a variety of NASA analogs prior to BASALT, including PLRP, Desert RATS, NEEMO and MVP $[4,5,9,10]$. The BASALT-1 deployment drove the design, development, and evaluation of new xGDS capabilities specific to supporting human EVA science operations and integration with Playbook and SEXTANT.

xGDS was designed to provide integrated support for science operations across all phases of a mission, with particular emphasis on pre-mission planning and data integration to support rapid science decision making during and after operations. xGDS supports the following four mission phases:

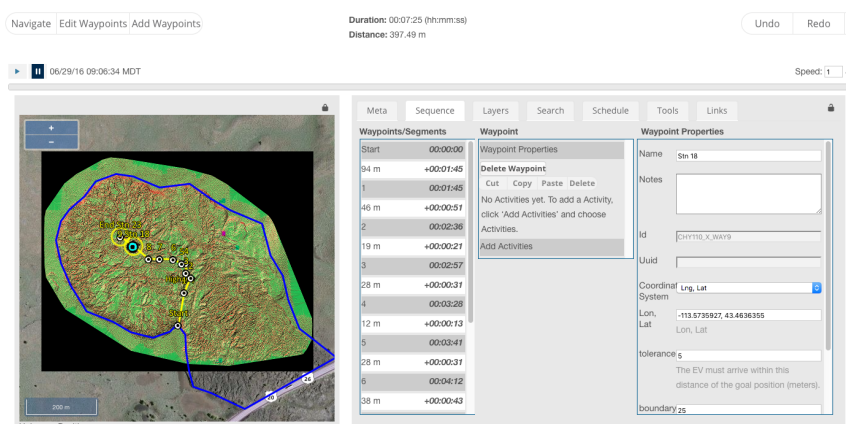

Figure 3. xGDS Traverse Plan editor
Planning - xGDS uses a-priori map information e.g. remote sensing data, known operational hazards or constraints, and targets of interest. xGDS enables teams to import, create and share map content and collaboratively edit EVA traverse plans, as shown in Figure 3.

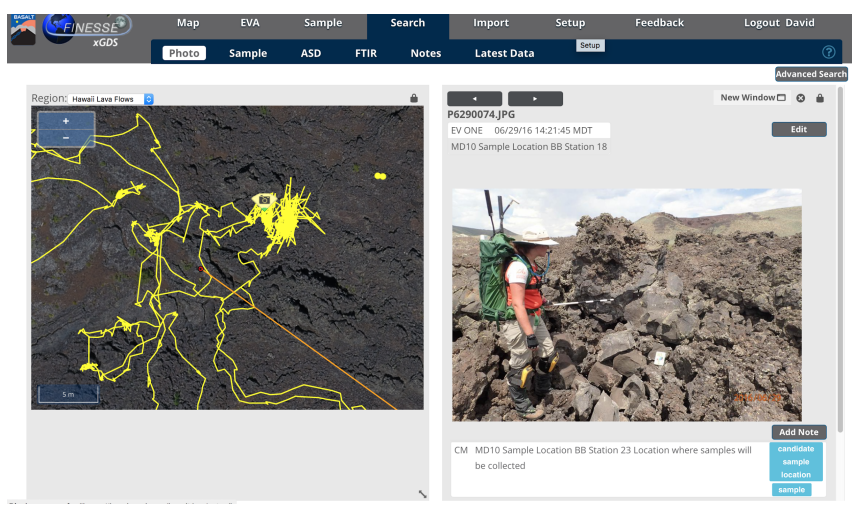

Figure 4. Monitoring crew on EVA; map with tracks on left, photo on right

Monitoring - xGDS ingests live telemetry data from field systems (e.g. GPS tracking) and generates maps, raster data overlays and 2D plots to visualize payload telemetry and EVA or vehicle position in real-time or simulated delay mode. It also includes facilities for real-time and post-hoc documentation and annotation, as shown in Figure 4.

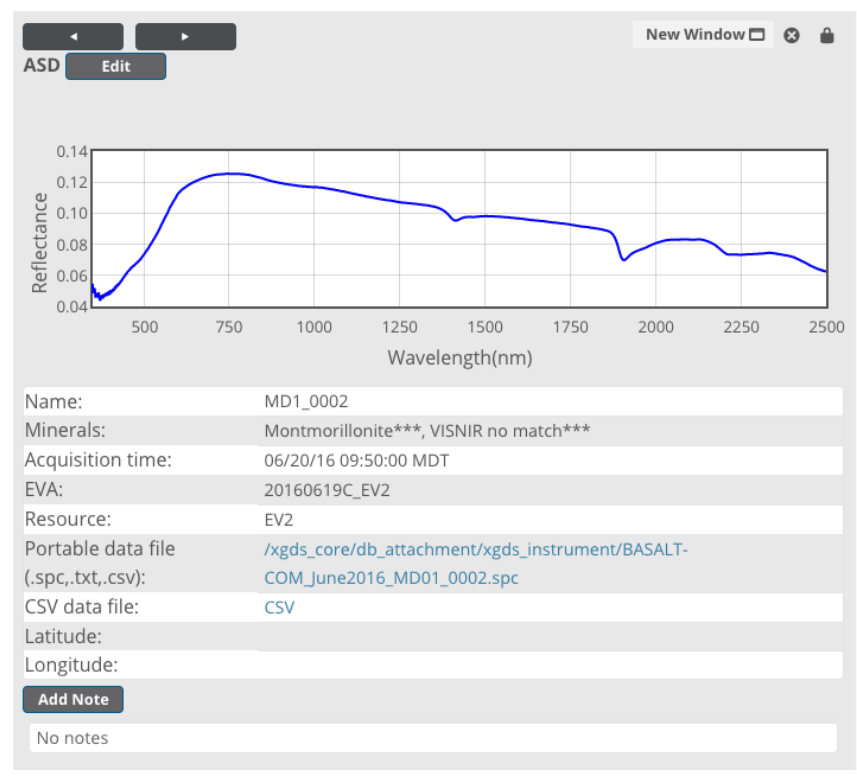

Figure 5. ASD instrument plot view

Archiving-As xGDS processes field telemetry, it reduces data to meaningful and efficient representations and organizes it into databases for later analysis, search, visualization, and export, as shown in Figure 5.

Exploring-xGDS provides capabilities to quickly explore collected data, see where and when it was collected, and search for data products and the correlations between them. Real-time semantic labeling greatly facilitates this 
functionality; xGDS users add notes and tags to data products and timelines in real-time during operations to support future search and analysis. Data search and visualization capabilities are used during and between EVA operations in a deployment (e.g. to select sampling sites or plan a day's activities) as well as post-deployment to support more detailed analysis.

\section{SEXTANT}

SEXTANT is a resource-based path planning tool that aims to optimize human traverses through terrain with elevation provided via data elevation models, applying different cost functions - traverse length, traverse time or metabolic rate[14]. SEXTANT also has the capability to optimize the concurrent paths of multiple humans and vehicles [11]. Although SEXTANT has previously been deployed in hypothetical case studies, BASALT-1 is the first time it has been tested in the field. In the context of BASALT, SEXTANT can be used to find optimal routes for the EV crew, from the EVA starting point through several waypoints.

SEXTANT offers four key functions: first, it establishes a guideline path that the IV team can use in order to monitor if the EV Crew are walking in the right direction towards the intended target. Second it can estimate path traversal times, which during mission planning enables the calculation of a better estimate on the time available for other activities. Third, during mission planning SEXTANT can determine whether some of the proposed sample locations are reachable or not. Lastly, during the EVA, the support personnel can ensure the EV Crew maintain an appropriate heading to reach a certain sampling location, easing the navigational demand on the EV Crew as they walk through the terrain.

Previous SEXTANT work has focused on the application of the tool to hypothetical Lunar EVA traverses; it was built to be general enough to apply to any planetary surface scenario. The architecture of SEXTANT is composed of three components: the terrain model, based on digital elevation maps (DEMs), the human or vehicle energy consumption model, which incorporates empirical velocity and energy consumption models depending on the slope of the terrain and gives rise to different cost functions, and a solver, which optimizes the cost function across the traverse. For BASALT, the terrain DEMs can range from one meter resolution based on stereo imagery from satellite images to on the order of centimeters resolution collected from drone sensors. The cost models are based on astronaut EVA suit test data, and as such are not accurate for predicting the energy consumption for the unsuited BASALT EVA crew because they overestimate energy consumption. Since the cost function for unsuited EVA with respect to the slope should follow a similar trend (just be of a smaller magnitude), optimization with the SEXTANT built-in cost functions should lead towards optimal paths for the EV Crew. Future work will focus on incorporating unsuited crew energy consumption.
SEXTANT is a numerical tool that produces an optimal traverse plan across a given terrain, such as shown in the example illustrated in Figure 6. The outputs include the planned traverse coordinates and timings. The original SEXTANT as developed by Johnson[14] also included a graphical user interface where the user could get visual feedback on the terrain, and the paths generated, displayed on top of a $3 \mathrm{D}$ relief map as shown in Figure 7. The interface also plotted elevation data and information on energy consumption throughout the traverse. For the initial incorporation of SEXTANT with xGDS these visualization features were eliminated, leaving the basic functionality of planning traverses as a black box, and adapting xGDS visual features.

\section{Integration and Work Flow}

Data flow between $x G D S$ and SEXTANT-SEXTANT's Python implementation adheres to a documented API. The SEXTANT code is deployed on the xGDS server. Once users have created an xGDS Planned Traverse, they click a button in the $x$ GDS plan editor to invoke SEXTANT. Users pass in constraints to optimize on energy, time or distance, control maximum slope and map resolution (or step granularity) that SEXTANT will use. The xGDS Planned Traverse is passed to SEXTANT in the xGDS xpJson format. SEXTANT reads the Planned Traverse's station coordinates out of the xpJson.

Once SEXTANT has finished processing, it returns Json data containing the original stations plus arrays of data between the Planned Traverse's stations. These are rendered as line segments in the xGDS plan editor, and the updated time estimates are reflected in the xGDS plan editor. When the Planned Traverse is exported to Google Earth via KML it includes these SEXTANT coordinates.

When users modify the planned traverse, they can clear and recalculate the SEXTANT generated paths.

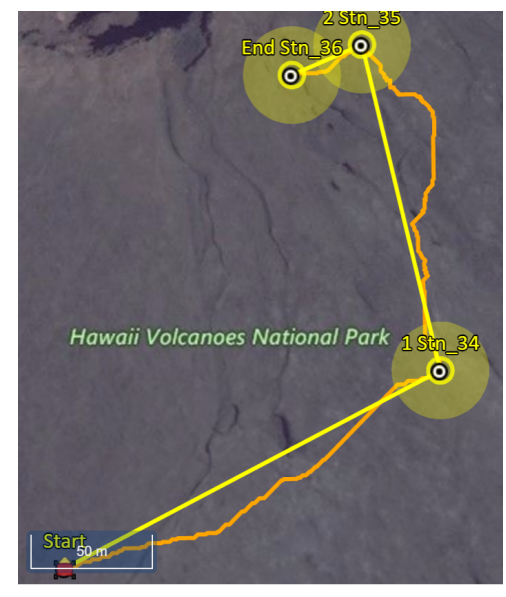

Figure 6. SEXTANT in the xGDS plan editor: waypoints are defined by a human planner, yellow lines are straight line path, orange lines show SEXTANTs optimized path using metabolic energy consumption as the cost function. The DEM has $0.5 \mathrm{~m}$ resolution. 


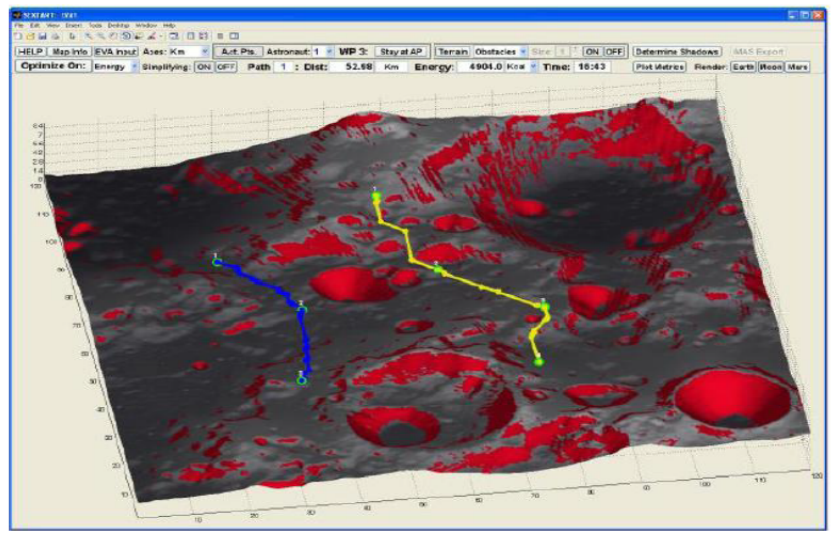

Figure 7. Original SEXTANT user interface designed for lunar path planning (from [14])

Data flow between $x G D S$ and Playbook-Within xGDS for each mission day an association is made between a crew member and a Planned Traverse, with an expected start time. The Planned Traverse can then be manually imported into Playbook. This includes the start times and durations for all of the waypoints within the Planned Traverse, any activities with their start times and durations, along with the duration estimates from SEXTANT if any, or straight line duration estimates.

Data flow between instruments, $x G D S$ and science teamTo support decision making during execution, xGDS gathers and displays live data from field systems. For BASALT, still images and various IR and XRF spectra are key data for science planning and guidance during EVAs. Unfortunately, most commercially available analytical instruments and cameras have undocumented, proprietary interfaces. We were able to overcome this limitation to some extent by using a Toshiba FlashAir W03 SD card for data storage in our field devices. This card is WiFi capable with an onboard scripting engine that supports HTTP data transfer and has a documented API. When a device saves a file to the SD card, an event is fired that initiates an HTTP data transfer to the xGDS server so that new images and data are immediately available to the science and operations teams.

\section{Field Testing}

The BASALT-1 simulation environment was designed to mimic the latest concept of operations proposed by the Evolvable Mars Campaign (EMC) and previous NASA analog research projects [5,8], see Ref. [15] for more detail concerning the operational details and study design. In summary, the BASALT-1 field deployment included an EV and IV crew comprised of two members each. While the IV team was temporally on Mars-time, they were physically located at the BASALT team encampment approximately 50 $\mathrm{km}$ away from the field site and the EV team. Specifically, the EV team was located within Craters of the Moon National Monument and Preserve (Idaho) and tasked with conducting science-driven EVAs. Each EVA was designed to be $\sim 4$ hours in duration and over the course of the 11-day field deployment, 10 EVAs were successfully conducted. For each EVA, the entire operations team was structured as shown in Figure 8 to conduct operations. Figure 8 also shows the Minerva users and their respective roles within the operations team. There were three main Minerva user groups: EV crew, IV crew and ST members. Two EV Crew utilized a portable, wrist mounted display to access xGDS for GPS tracking and Playbook for mission log messaging. Two IV Crew members collectively accessed and interfaced with all components of Minerva from console workstations that were collocated with the EV (e.g. no time-delay between EV/IV communication). The ST (10-15 members) utilized all components of Minerva in a distributed manner where the different features/capabilities of xGDS were delineated to specific roles within the ST, as described in Table 1. Situated within the MSC was a large monitor that projected video and GPS data for the entire ST team to view and track EV crew activities.

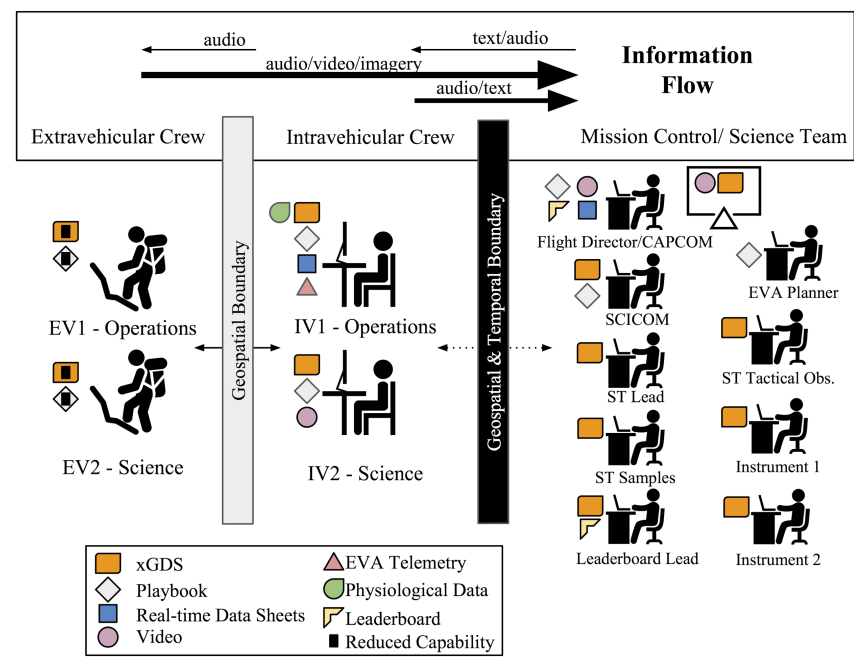

Figure 8. BASALT-1 field test setup

The information shared between the operational units varied in content and relay direction and represent a fundamental change from present-day information flow between MSC and flight crew $[16,17]$. For example, all data from the EV crew streamed locally to the IV operators and to the ST (accounting for both 5 and 15 minute one-way light time delays, depending on the test condition). The IV crew conveyed real-time audio/GPS data to the EV crew. The ST was only capable of sending audio and text information to the IV crew. Previous NASA analog studies have concluded that audio communication sent from ST to EV crew over time-delayed communication is distracting and has limited overall utility. Instead, all ST input was directed to the IV so they could synthesize ST input and convey relevant data/instruction to the EV crew in a more appropriate (e.g. timely and contextually relevant) manner. 
Table 1. Roles and Responsibilities

\begin{tabular}{|c|c|c|}
\hline Unit & Name & Responsibilities \\
\hline \multirow{2}{*}{ EV } & EV1 & $\begin{array}{l}\text { Execute EVA timeline tasks and provide operational } \\
\text { insight regarding timeline, flight rules/constraints, and } \\
\text { procedures }\end{array}$ \\
\hline & EV2 & $\begin{array}{l}\text { Execute EVA timeline tasks and provide scientific insight } \\
\text { based on scientific expertise (e.g. geologist, geochemist, } \\
\text { etc.) }\end{array}$ \\
\hline \multirow{2}{*}{ IV } & IV1 & $\begin{array}{l}\text { Main interface between CAPCOM and EV1 \& 2; guide } \\
\text { EV1 \& } 2 \text { based on EVA timeline, flight rules/constraints, } \\
\text { and procedures }\end{array}$ \\
\hline & IV2 & $\begin{array}{l}\text { Main interface between SCICOM and EV1 \& 2; interact } \\
\text { with EV1 \& } 2 \text { about science tasks, priorities, and } \\
\text { procedures based on ground ST input }\end{array}$ \\
\hline \multirow{9}{*}{$\mathrm{MCC} / \mathrm{ST}$} & $\begin{array}{l}\text { Flight } \\
\text { Director } \\
\text { /CAPCOM }\end{array}$ & $\begin{array}{l}\text { Responsible for strategic-level EVA decisions related to } \\
\text { prioritization of tasks, science, etc.; ensure flight rules are } \\
\text { being followed, coordinates with IV1 }\end{array}$ \\
\hline & $\begin{array}{l}\text { EVA } \\
\text { Planner }\end{array}$ & $\begin{array}{l}\text { Execute timeline and traverse changes in Minerva during } \\
\text { EVAs }\end{array}$ \\
\hline & SCICOM & $\begin{array}{l}\text { Help ST maintain operational situational awareness } \\
\text { throughout the EVA (e.g., carefully track EVA timeline } \\
\text { noting deviations by crew, inform ST when feedback must } \\
\text { be relayed to crew, etc.) } \\
\text { Coordinate with ST Lead to relay ST input on science } \\
\text { tasks, priorities, and procedures to IV crew } \\
\text { Coordinate with Flight Director on EVA extension requests }\end{array}$ \\
\hline & ST Lead & $\begin{array}{l}\text { Maintain general science situational awareness throughout } \\
\text { the EVA to ensure that EVA's science objectives are met; } \\
\text { lead the ST in providing tactical feedback to the EV/IV } \\
\text { crew to guide the current EVA; work with SCICOM to } \\
\text { relay plans/recommendations to the crew }\end{array}$ \\
\hline & ST Samples & $\begin{array}{l}\text { Analyze science from EV crew compared to precursor } \\
\text { planned science traverses, interact w/ ST lead on science } \\
\text { input to provide to IV/EV }\end{array}$ \\
\hline & $\begin{array}{l}\text { Leaderboard } \\
\text { Lead }\end{array}$ & $\begin{array}{l}\text { Maintain accurate record of collective ST input regarding } \\
\text { sample prioritization }\end{array}$ \\
\hline & $\begin{array}{l}\text { ST Tactical } \\
\text { Obs. }\end{array}$ & $\begin{array}{l}\text { Focus on incoming information from EV/IV crew (voice, } \\
\text { video, still imagery, science data) and provide tactical } \\
\text { input to guide future tasks within the current EVA }\end{array}$ \\
\hline & Instrument 1 & $\begin{array}{l}\text { Focus on incoming instrument-specific data from EV/IV } \\
\text { crew and provide tactical input to guide future tasks within } \\
\text { the current EVA }\end{array}$ \\
\hline & Instrument 2 & $\begin{array}{l}\text { Focus on incoming instrument-specific data from EV/IV } \\
\text { crew and provide tactical input to guide future tasks within } \\
\text { the current EVA }\end{array}$ \\
\hline
\end{tabular}

Table 1 shows a brief description of each responsibility delegated to each member of the operations team. One main research question for BASALT is how to best structure and support ST operations to actively influence EVA execution across time-delay. That is, how can a team of scientific experts download, synthesize, analyze, and generate scientific feedback to ensure EVA productivity and mission success? A defining characteristic of scientifically driven EVA operations is that to truly make progress towards scientific objectives involves an iterative scientific thought process with typically ill-determined or unpredicted success criteria. Contrary to ISS EVA operations where task success is clearly defined by hardware configurations and system states, the EVAs performed in BASALT require a scientific understanding of EV/IV crew activities to ensure successful progress is being made toward the overall science objectives. For a brief review of the science objectives for BASALT-1 see Ref. [15]. As discussed in subsequent sections, we experienced various degrees of success regarding supporting this science synthesis process using Minerva and uncovered additional use-case perspectives.

The adopted field operations protocol for BASALT-1 was to support two main channels of communication, one that emphasizes task execution, timeline, and flight rules/constraints management (Operations) and one that focuses on scientific discussion and development (Science). As described in Table 1, the IV operators played a critical role in the interface between ST input, EV input and their own perspectives based on the aggregated data presented to them. The IV acted as a relay point of information and input from the EV and ST to instruct and guide EV task execution. Within the ST, the capsule communication (CAPCOM) provided operations relevant input which consisted primarily of time management and flight rules content. The science communicator (SCICOM) conveyed a highly condensed summary of ST survey and sampling priorities as well as scientific justification and rationale throughout each EVA.

\section{Table 2. Subset of BASALT-1 use cases with associated software capabilities}

\begin{tabular}{|c|c|c|c|c|}
\hline \multirow{2}{*}{\begin{tabular}{|c} 
Mission \\
Phase
\end{tabular}} & \multirow[b]{2}{*}{ Use Case Description } & \multicolumn{3}{|c|}{ Software (Minerva) } \\
\hline & & xGDS & Playbook & Sextant \\
\hline \multirow{2}{*}{$\begin{array}{c}\text { Pre- } \\
\text { Mission }\end{array}$} & Create exploration traverse & $\checkmark$ & & $\checkmark$ \\
\hline & $\begin{array}{l}\text { Create science plan for } \\
\text { station }\end{array}$ & $\checkmark$ & $\checkmark$ & \\
\hline \multirow{5}{*}{$\begin{array}{l}\text { Intra- } \\
\text { Mission }\end{array}$} & $\begin{array}{l}\text { Propose change to science } \\
\text { plan for station }\end{array}$ & $\checkmark$ & $\checkmark$ & $\checkmark$ \\
\hline & $\begin{array}{l}\text { Modify science plan for } \\
\text { station }\end{array}$ & $\sqrt{ }$ & & \\
\hline & $\begin{array}{l}\text { Provide science team input } \\
\text { to crew }\end{array}$ & $\sqrt{ }$ & $\checkmark$ & \\
\hline & Modify planned traverse & $\checkmark$ & & $\checkmark$ \\
\hline & $\begin{array}{l}\text { Provide planned traverse } \\
\text { input to crew }\end{array}$ & $\sqrt{ }$ & & $\checkmark$ \\
\hline \multirow{4}{*}{$\begin{array}{l}\text { Post- } \\
\text { Mission }\end{array}$} & $\begin{array}{l}\text { Analyze as-run traverse \& } \\
\text { science data }\end{array}$ & $\checkmark$ & & \\
\hline & $\begin{array}{l}\text { Analyze data during EVA } \\
\text { to high-grade samples }\end{array}$ & $\checkmark$ & & \\
\hline & $\begin{array}{l}\text { Sample w/ post-processed } \\
\text { instrument Inter-EVA }\end{array}$ & $\checkmark$ & & \\
\hline & $\begin{array}{l}\text { Analyze sample data after } \\
\text { field season }\end{array}$ & $\checkmark$ & & \\
\hline
\end{tabular}


Use Cases-Early in the development stages of the BASALT project, a use case development exercise was performed $[18,19]$. The intent of these activities was to articulate what necessary capabilities and functions Minerva would need to provide to ensure a successful field deployment, similar to efforts in other complex work domains such as health care [20], spaceflight operations [21,22], and air traffic operations [23]. As shown in Table 2, Minerva was expected to provide operational support in all facets of the deployment: pre-EVA, intra-EVA, and postEVA. The xGDS and Playbook software packages have had extensive use in the field as previously mentioned. The challenge for BASALT-1 was to begin integrating each tool's capabilities to ensure adequate capability coverage while minimizing duplication of resources.

Two primary pre-EVA use cases identified were concerned with EVA timeline development in the form of a planned traverse route and a science plan for each station along the route. The ST collected a host of scientific data about the field site prior to the deployment to build a contextual understanding of what to expect to encounter while in the field. This precursor data was used to inform the creation of traverse plans and science plans to be executed by the EV/IV flight team. The intra-EVA activities Minerva was intended to support included modifying the science plan and traverse plan and facilitating the synthesis of scientific data to be conveyed to the relevant operational members. The synthesis of science data included the ability for the ST and IV to take notes directly associated with the streaming video and still imagery obtained by the EV Crew. The various console positions within the ST provided context specific notes depending on their expertise with the observed data (e.g. the Instrument ST members added instrument-specific insight in the form of notes in xGDS). Additionally, xGDS enabled the tagging and association of geospatial data to sample imagery and video to enable the ST to search and filter for specific samples. Playbook provided the ability to convey condensed ST input and feedback via the Mission Log. Once the EVA was completed, xGDS provided data archiving and post-processing support. These use-cases were experienced heavily during the field deployment to include relevant data that might have been missed during the EVA and provide an aggregate dataset to compare to the science objectives to inform subsequent planning activities.

As shown in Table 2, some use cases are supported by multiple tools in the Minerva suite. While it may appear redundant to have multiple tools support the same use case, in practice each tool has specific strengths for the given shared use case. For example, for creating and proposing changes to a science plan for a station, xGDS supports geospatial based planning, Playbook supports activity based planning, and SEXTANT assists in human traverse planning. While there was some integration between the tools for BASALT-1 we hope to further integrate the tools for these shared use cases in future BASALT deployments.
Emergent Use Cases-As is typical with complex operations, particularly with envisioned work environments, we experienced some emergent use cases that played a critical role in the execution of BASALT-1 that had not been previously identified. Specifically, we highlight four use cases observed among the ST that will be explored in future deployments.

Outside of the Minerva software previously described, the ST leveraged a digital leaderboard to help manage the tactical preferences and priorities of the samples viewed and sampled during each EVA, as shown in Figure 9. Using the Google sheets infrastructure, the ST was able to distill the detailed notes and scientific data being recorded by xGDS to summarize the candidate samples. This capability was dynamically changing throughout the EVA as more data was obtained by the ST, which then influenced scientist preferences and opinions about how the samples comparatively ranked against each other. The distributed nature of the ST necessitated a console position dedicated to the management of the leaderboard so that a consistent version existed. Given their close physical proximity to each other, the scientists often collectively discussed the state of the leaderboard and collectively made alterations to the rank order in an evidence based manner by viewing the data presented in xGDS.

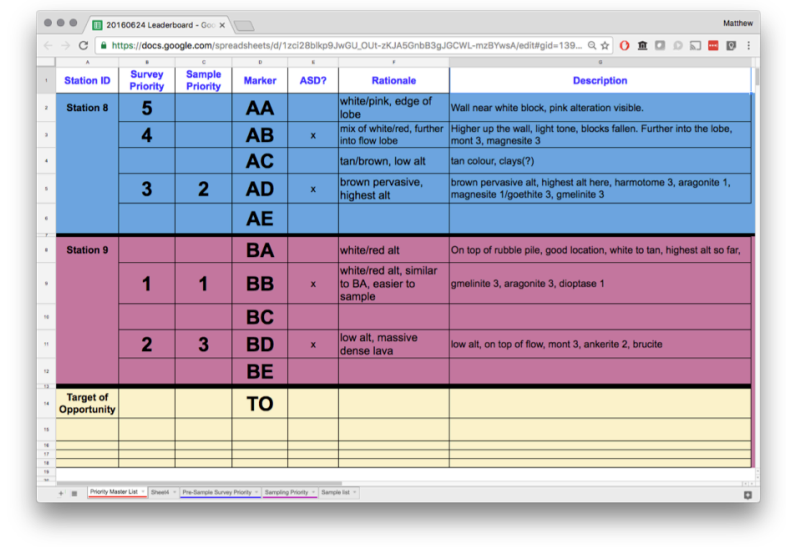

\section{Figure 9. An improvised "leaderboard" capability was used by the science team to maintain an up-to- date list of priorities during each EVA.}

In addition to the leaderboard to manage tactical preferences, the ST employed a science data matrix to manage strategic data and decisions that directly linked to the underlying scientific objectives. Between EVAs, the scientist would update the relevant sections of this science matrix to help track and maintain awareness of overall progress towards each research question. As experienced in previous scientific field operations, the detection and quantification of scientific research progress is a highly iterative process [5,24]. Therefore, quantifying successful research progress can be difficult. At first glance, the count and type of samples collected provide a quantifiable metric 
by which progress can be measured, but only after detailed analysis of those samples can a more complete picture of scientific understanding be measured. The level of detail and 'minimum success criteria' specified in the scientific research questions can be somewhat ill-defined, depending on the scope and aim of the question, which then influences the operational details necessary to plan and include in the EVA timeline.

The BASALT-1 deployment executed 1 EVA per $\sim 24$ hours which provided time for the ST to preliminarily postprocess scientific data to influence subsequent EVAs. A major use case of the xGDS software was its ability to provide archived data to the ST when they were formulating their EVA objectives. A general trend observed during the deployment was that replanning occurred frequently between EVAs, when the scientists had prolonged discussions about the collected data, while replanning was infrequent during the execution of EVAs. This observation was in part due to the standardized study design timeline imposed by the operations research objectives. There was evidence of confusion in the timeline when reprioritization of samples was conveyed, particularly when sent in rapid succession from the ST. The EV/IV operate on a separate temporal timeline from the ST; by the time the ST downloaded, processed and sent feedback, the EV/IV crew have moved beyond the content and attention of the content to which those feedback messages pertain. In cases where multiple prioritization messages were sent, the ST also provided justification to help the IV crew understand ST rationale.

A final use case that emerged from the ST during BASALT1 pertained to the distinction between science discussion and general operational awareness. Often the ST would become immersed in discussion regarding particular aspects of samples and lose awareness of what the EV crew were doing, necessitating another operator to brief the team when people wanted to know what was currently being executed. The large video and GPS tracking monitor provided access to this detail for all ST members in terms of what the crew were visually seeing and doing and where they were. The ST also wanted to know if any additional samples had been identified and sample specific details. The Playbook timeline provided an overall timeline view of the asperformed progress of the EV crew, but not sample-specific details. While the xGDS tool provided a comprehensive data input platform, the ability to quickly synthesize timeline progress was difficult to discern, particularly given the volume of data xGDS presented in real-time.

\section{Results}

The BASALT-1 field test in Idaho provided an opportunity to conduct an initial evaluation of Minerva, focused mainly on usability. We currently lack preliminary field-test evaluation for SEXTANT (see Lessons Learned), but we surveyed all participating team members with regards to the usability of Playbook and xGDS.
Playbook preliminary evaluation-We received positive feedback for the real-time Timeline and Mission Log capabilities. In particular, team members noted liking EVA phase statusing (e.g., knowing which EVA phase was happening at that moment) and the additional Mission Log messages' cue for time to delivery and first possible response. One BASALT field member noted: "Playbook enabled me to better manage my time and attention during high intensity periods [of work]." In terms of areas of improvement, we identified work processes and capabilities that warrant attention. A tighter workflow between ST planning with xGDS and Playbook could increase efficiencies and minimize miscommunications. With regards to Playbook, additional countermeasures or capabilities are needed to more effectively communicate over periods of high message exchange and long latencies. Field team members requested capabilities such as chat threads and marking critical or important messages.

$x G D S$ preliminary evaluation-We received positive feedback on spatial integration of data products, live EVA tracking, ease of traverse planning, and centralized storage and sharing of data. In terms of areas of improvement, we identified requests for more flexibility in user interface layout, likely depending on roles and responsibilities, improvements to real-time data delivery and requests for improved or streamlined search and tagging capabilities.

During BASALT-1, a significant portion of the crew, Mission Support and Science teams had limited experience using Minerva. Some had previous exposure to xGDS or Playbook, but rarely both; SEXTANT is a new capability to all team members. As the team becomes more experienced and familiar with the toolset, future evaluations will focus on the utility of Minerva as an enabler for Mars EVA. Specifically, we will look at communication content and structure, communication messages relating to the EVA timeline, specific capability assessments (as defined by [15] as part of larger consensus ratings, and user interaction assessments. Additional measures and operational metrics are currently being devised and assessed to help quantify the evaluation of Minerva. The EVA work domain currently has limited standard evaluation methodologies to examine operations, therefore as part of the Minerva development effort, a concerted effort needs to be made to define what measures are relevant and valuable to describe this complex operational environment.

Lessons Learned-Three major lessons were derived from BASALT-1 which include: 1) better integration of timeline and procedure views from Playbook, 2) enhancing high rate of text communication exchanges over time-delay, and 3) enhancing traverse path routes. Additional perspectives will be explored in subsequent publications.

Playbook broadcasts timeline changes and status of the activities in the Timeline view collaboratively so that the whole team sees the same information. During BASALT-1, the EVA Planner statused activities as in-progress or complete and changed the start time of the activities to 
reflect the as-run plan, but the duration of the activities was fixed. During execution, we discovered that the duration of an activity highly depends on the maneuverability of terrain and samples priorities by the ST, e.g. time to extract samples, traverse between samples, etc. When start times and durations of activities changed, the visual representation of the timeline shifted to overlap activities which wrongly conveyed simultaneous task execution. Allowing the status and duration of the activities in Playbook to change, to better reflect the EVA as it is being executed, will give the Mission Support Center an at-a-glance situation awareness directly from the timeline. Ideally, the crew would update the status of the activities so the ground would receive that information after the elapsed time-delay, rather than independently updating their own timeline. As for procedures, the BASALT team creates a vast collection of documents, flight rules, and meeting briefs and handovers that exist in their own repository, accessed independently and distributed via email. Playbook activities can link to documents to give users direct access to them, and better awareness of the procedures they need to review.

Because BASALT operates under either 5 or 15-minute time-delay communications with the flight crew, the ST must often make decisions with incomplete or partial information (e.g. video, audio, imagery) to provide EV/IV crew with ST preferences. As new information is received, updates to the previous science priorities can be generated and conveyed to the EV/IV team via the Playbook Mission Log. Subsequently, numerous messages can be 'in-transit' to the EV/IV crew any one point in time, depending on how quickly ST input/feedback is generated. This backlog of messages causes the messages to arrive in rapid succession from the IV perspective, creating a period of heavy workload that can cause the crew to miss messages. Furthermore, within the exchange of text messages is the challenge of confirming that messages were successfully received and understood. A method to quickly acknowledge message receipt and comprehension could promote a greater shared situation awareness among the flight personnel. Finally, the content of the messages should dictate a specific prioritization within the chat client. For the next BASALT deployment, high priority messages will be introduced to force messages to remain at the top of the chat client until dismissed, to ensure important messages are read.

Having correct traverse plans that contour obstacles and follow less steep inclines ahead of the EVAs provide a significant advantage, as incorrect plans can lead to the EV crew having to independently reassess and plan their paths during a traverse. This was demonstrated when SEXTANT had to be replaced with manual path planning due to map resolution limitations at $10-15 \mathrm{~m}$ : the original guiding waypoints for the EV crew to get to the sampling locations sent the crew through too treacherous and abrupt terrain, which they only discovered during the traverse. This lead to real-time manual replanning, ultimately requiring extra time resources (and in a real EVA environment this would have translated to additional use of consumable resources such as oxygen). The offset between the guidance waypoints and the path taken by the crew is shown in Figure 10.

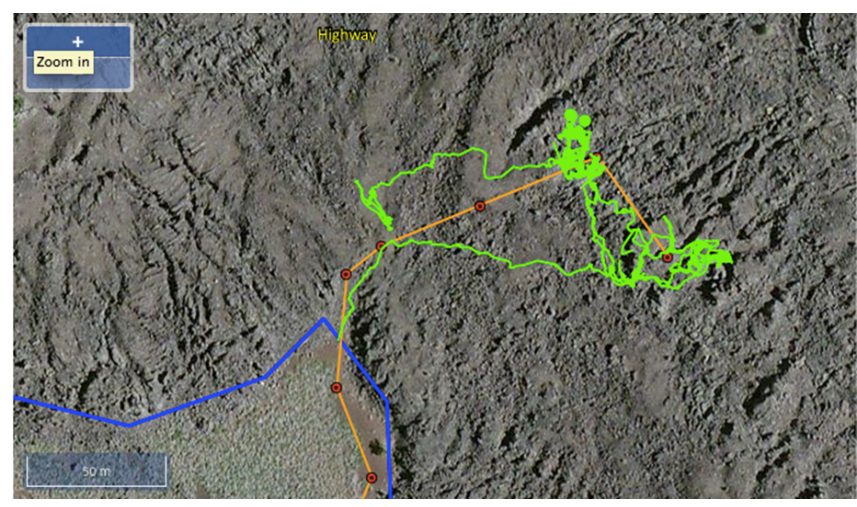

Figure 10. Comparison of manually planned EVA route (orange) and actual route (green)

This stands in contrast with the plan developed with high resolution data $(\sim 3 \mathrm{~cm})$. Although the guiding waypoints were not generated by SEXTANT, but by the user, Figure 11 shows how well the GPS tracks stick to the guiding waypoints, confirming the quality of the path taken, and thus staying on schedule.

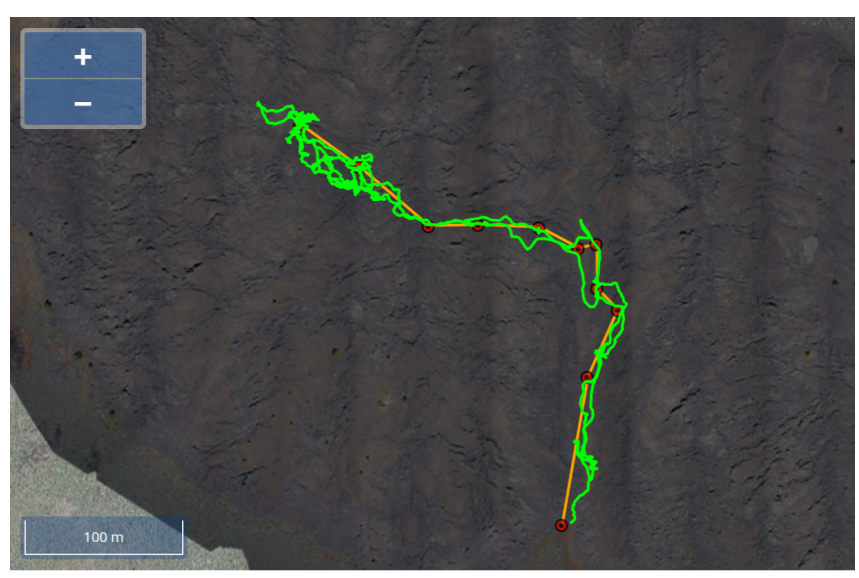

Figure 11. Manually planned waypoints, with high resolution $(3 \mathrm{~cm})$ data elevation map. Green indicate flatter areas, red indicates steeper areas. Waypoints in red, actual GPS track in bright green.

In these cases the planning was done manually. SEXTANT automates the process, saving significant workload. Additionally, SEXTANT is expected to return easier plans for the EV crew to traverse, as the energy consumption for the crew can be optimized, a feature manual planning does not offer. SEXTANT is challenged by the large amount of data it needs to parse to find paths through high resolution maps, and the lack of ability to generate any useful path when relying on low resolution maps. Current work is focusing on assessing what middle-ground resolution would be optimal for SEXTANT to perform well in abrupt terrain such as the Idaho lava flow. 


\section{FUTURE WORK}

The BASALT project will have additional field deployments, where we will continue to improve on Minerva's capabilities and BASALT workflows. Of particular importance is to further integrate the toolsets. We have begun doing this with xGDS and SEXTANT, with an initial focus on performance optimization and improvements to the mechanics of systems integration. In the future, we will investigate dynamic user displays of SEXTANT results to give planners richer information and greater confidence in the routes planned by SEXTANT. We are also building additional features into Playbook communication and flight following capabilities based on our observations from BASALT-1.

In a previous analog mission, xGDS and Playbook shared scheduled activities, where the duration of activities planned for sites were imported into Playbook as a timeline. Future integrated capabilities will likely include bringing this capability to BASALT deployments, and sharing live location and activity status in order to provide an EVA status board, where ground teams could quickly see what is happening at the moment during the EVA in an integrated temporal and spatial view of EVA activities.

Future Playbook functionality also includes highlighting high priority Mission Log messages, searching Mission Log messages, activities with modifiable durations, and improved server connectivity.

Future xGDS development and assessment will likely include:

- Improved real-time data delivery and live event notification for web clients.

- Improved search and semantic tagging tools.

- Continued improvements to human EVA specific capabilities (vs. robotic assets).

- Working with Playbook and SEXTANT teams on tighter integration of capabilities.

Ongoing assessment with new and experienced users as addition capabilities are developed and deployed

\section{ACKNOWLEDGEMENTS}

BASALT is funded by the NASA Planetary Science and Technology Through Analog Research (PSTAR) Program (NNH14ZDA001N-PSTAR) grant (14-PSTAR14_2-0007) to D. Lim. Coauthor M. Miller is supported by a NASA Space Technology Research Fellowship (NNX13AL32H). Some additional support comes through collaboration with the NASA Solar System Exploration Research Virtual Institute (SSERVI) Field Investigations for Solar System Exploration (FINESSE) grant to J. Heldmann. In addition, the authors would like to thank Craters of the Moon National Monument \& Preserve (Permit \# CRMO-2014SCI-0004), Hawai'i Volcanoes National Park (Permit \#HAVO-2016-SCI-0023), The community of Arco, Idaho and the staff at COTM, and the Kilauea Military Camp (KMC).

\section{REFERENCES}

[1] D. A. Craig, N. B. Herrmann, and P. A. Troutman, "The Evolvable Mars Campaign - study status," presented at the 2015 IEEE Aerospace Conference, 2015, pp. 1-14.

[2] B. G. Drake, Ed., "Human Exploration of Mars Design Reference Architecture 5.0 - Addendum," Mars Architecture Steering Group - NASA Headquarters, NASA/SP-2009-566-ADD, Jul. 2009.

[3] B. G. Drake, Ed., "Human Exploration of Mars Design Reference Architecture 5.0," NASA Headquarters, NASA/SP-2009-566, Jul. 2009.

[4] D. S. S. Lim, A. L. Brady, A. F. Abercromby, D. T. Andersen, et al., "A historical overview of the Pavilion Lake Research Project-Analog science and exploration in an underwater environment," vol. 483, pp. 85-115, 2011.

[5] M. J. Miller, D. S. S. Lim, A. L. Brady, Z. Cardman, E. R. Bell, W. B. Garry, D. Reid, S. P. Chappell, and A. Abercromby, "PLRP-3: Conducting ScienceDriven Extravehicular Activity with Communications Latency," presented at the IEEE Aerospace Conference, 2016.

[6] S. P. Chappell, A. F. Abercromby, and M. L. Gernhardt, "NEEMO 15: Evaluation of Human Exploration Systems for Near-Earth Asteroids," Acta Astronautica, vol. 89, no. C, pp. 166-178, Mar. 2013.

[7] S. P. Chappell, A. F. Abercromby, M. Reagan, M. L. Gernhardt, and W. Todd, "NEEMO 16: Evaluation of Systems for Human Exploration of Near-Earth Asteroids," presented at the 43rd International Conference on Environmental Systems, Vail, CO, 2013.

[8] S. P. Chappell, K. Beaton, M. J. Miller, C. Halcon, G. Michael, and A. Abercromby, "NEEMO 18-20: Analog Testing for Mitigation of Communication Latency during Human Space Exploration," presented at the IEEE Aerospace Conference, 2016.

[9] J. L. Heldmann, A. Colaprete, A. Cook, T. Roush, M. Deans, R. Elphic, D. Lim, J. R. Skok, N. E. Button, S. Karunatillake, and G. Garcia, "Mojave Volatiles Prospector (MVP): Science and Operations Results from a Lunar Polar Rover Analog Field Campaign," presented at the 46th Lunar and Planetary Science Conferece, 2015.

[10] S. Y. Lee, D. Lees, T. Cohen, M. Allan, M. Deans, T. Morse, E. Park, and T. Smith, "Acta Astronautica," Acta Astronautica, vol. 90, no. 2, pp. 268-288, Oct. 2013.

[11] J. J. Marquez and M. L. Cummings, "Design and Evaluation of Path Planning Decision Support for Planetary Surface Exploration," Journal of Aerospace Computing, Information, and Communication, vol. 5, no. 3, pp. 57-71, Mar. 2008.

[12] E. J. Speyerer, S. J. Lawrence, J. D. Stopar, P. G. ser, M. S. Robinson, and B. L. Jolliff, "Optimized traverse planning for future polar prospectors based on lunar topography," pp. 1-9, Apr. 2016. 
[13] J. Marquez, G. Pyrzak, S. Hashemi, S. Ahmed, K. McMillin, J. Medwid, D. Chen, and E. Hurtle, "Supporting Real-Time Operations and Execution through Timeline and Scheduling Aids," presented at the 43rd International Conference on Environmental Systems, 2013, pp. 1-11.

[14] A. W. Johnson, J. A. Hoffman, D. J. Newman, E. M. Mazarico, and M. T. Zuber, "An Integrated Traverse Planner and Analysis Tool for Planetary Exploration," presented at the AIAA SPACE 2010 Conference \& Exposition, 2010, pp. 1-28.

[15] K. Beaton, S. Chappell, M. Miller, D. Lim, A. Abercromby, S. Kobs Nawotniak, A. Brady, and S. Hughes, "Extravehicular Activity Operations Concepts under Communication Latency and Bandwidth Constraints," 2017.

[16] M. J. Miller, K. M. McGuire, and K. M. Feigh, "Information Flow Model of Human Extravehicular Activity," presented at the In Proceedings of the IEEE Aerospace Conference, Big Sky, MT, 2015.

[17] M. J. Miller, K. M. McGuire, and K. M. Feigh, "Decision Support System Requirements Definition for Human Extravehicular Activity using Cognitive Work Analysis," Journal of Cognitive Engineering and Decision Making, 2016.

[18] J. M. Carroll, Making Use - Scenario-based design of human-computer interactions. Massachusetts Institute of Technology, 2000.

[19] M. B. Rosson and J. M. Carroll, "Scenario-Based Design," in The Human-Computer Interaction Handbook: Fundamentals, Evolving Technologies and Emerging Applications, no. 53, J. A. Jacko and A. Sears, Eds. Lawrence Erlbaum Associates, 2002, pp. 1032-1050.

[20] S. Z. Lowry, M. Ramaiah, S. Taylor, E. S. Patterson, S. S. Prettyman, D. Simmons, D. Brick, P. Latkany, and M. C. Gibbons, "Technical Evaluation, Testing, and Validation of the Usability of Electronic Health Records: Empirically Based Use Cases for Validating Safety-Enhanced Usability and Guidelines for Standardization ," US Department of Commerce, NISTIR 7804-1, Mar. 2016.

[21] D. Billman, L. Arsintescu, M. Feary, J. Lee, and R. Tiwary, "Needs Analysis and Technology Evaluation: Evaluation Case Study of Alternative Software for Controller Planning Work-Part 2," Journal of Cognitive Engineering and Decision Making, vol. 9, no. 2, pp. 186-207, May 2015.

[22] D. Billman, M. Feary, D. Schreckenghost, and L. Sherry, "Needs Analysis and Technology Alignment Method: A Case Study of Planning Work in an International Space Station Controller Group-Part 1," Journal of Cognitive Engineering and Decision Making, vol. 9, no. 2, pp. 169-185, May 2015.

[23] J. Cahill, N. McDonald, R. Morrison, and D. Lynch, "The operational validation of new cockpit technologies supporting all conditions operations: a case study," Cognition, Technology \& Work, pp. 131, Jun. 2016
[24] R. A. Yingst, P. Russell, I. L. ten Kate, S. Noble, T. Graff, L. D. Graham, and D. Eppler, "Designing remote operations strategies to optimize science mission goals: Lessons learned from the Moon Mars Analog Mission Activities Mauna Kea 2012 field test," Acta Astronautica, vol. 113, no. C, pp. 120 131, Aug. 2015.

\section{BIOGRAPHY}

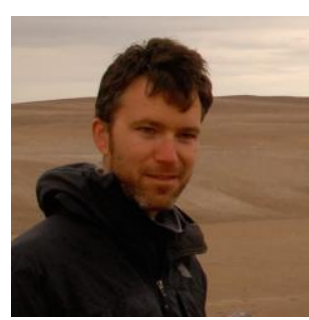

Matthew Deans received a Ph.D. in Robotics from Carnegie Mellon University in 2005. He works with the Intelligent Robotics Group at NASA Ames Research Center, researching robotic navigation, control, humanrobotic interfaces, and mission operations tools. He has been involved with numerous terrestrial analogs for planetary rovers and human missions to space, including field work in Antarctica and the Arctic, Chile, Hawaii, and many deserts in the continental US.

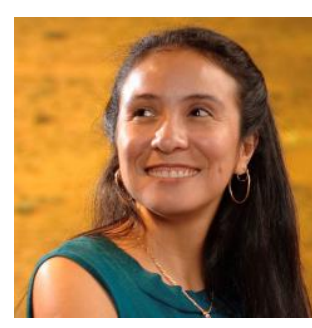

Jessica J. Marquez received a B.S.E. in Mechanical Engineering from Princeton University, followed by a S.M. from the Department of Aeronautics and Astronautics at MIT. She received her Ph.D. in Human Systems Engineering from MIT. Since 2007, she has been working at the NASA Ames Research Center within the Human Systems Integration Division where she has supported the development and deployment of various instances of SPIFe (Scheduling \& Planning Interface For exploration), the planning and scheduling software tool for several space missions including ISS. As the Human Research Program's Discipline Science for the Risk of Inadequate Design of Human and Automation/Robotics Integration, she continues to conduct research in the field of human-automation integration, human-computer interaction, and space human factors engineering.

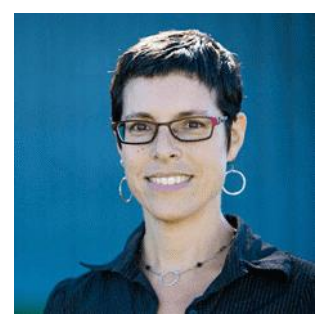

Tamar Cohen received a B.S. in Computer Science from Cornell University, followed by an M.A. in Fine Art / Visual Information Technology from George Mason University. She works with NASA Ames' Intelligent Robotics group to bridge the gap between robots and humans. She designs and implements software to provide situational awareness for remote robotic operation. She is a developer of $x G D S$ (Exploration Ground Data Systems) software, helping scientists plan, monitor and explore field data on a map. She previously worked on VERVE, an Eclipse based $3 D$ visualization toolkit. 


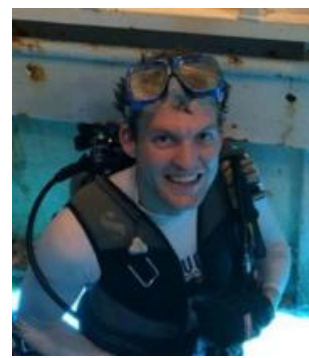

Matthew James Miller is a graduate research assistant in the Cognitive Engineering Center at the Georgia Institute of Technology. He has interned at the NASA Johnson Space Center, Charles S. Draper Laboratory, The Aerospace Corporation, and ATA Engineering. He received a B.S. and a M.S. in Aerospace Engineering from Georgia Institute of Technology in 2012 and 2014, respectively, and is currently pursuing his Ph.D. His research interests are in the areas of decision making and decision support development in complex work domains. He is supported by a NASA Space Technology Research Fellowship and is advised by Dr. Karen Feigh.

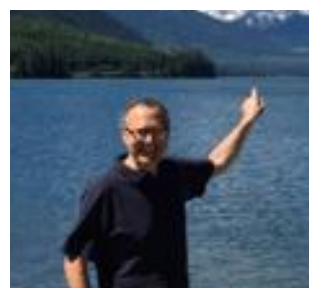

David Lees is a member of the Intelligent Robotics Group at NASA Ames and a Senior Project Scientist at Carnegie Mellon University's Silicon Valley campus. His research background is in human-robotic interaction and interfaces for terrestrial and space applications. While at Ames, he has supported the development, study and deployment of ground systems tools at multiple NASA field analogs. He has also developed and deployed $3 D$ visualization tools to support the MER and MSL Mars missions. He received a $B S$ degree in EECS from Johns Hopkins University, followed by and MS and Ph.D. in Mechanical Engineering from Stanford University.

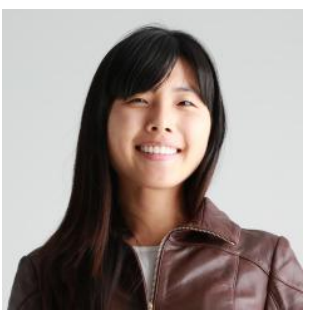

Yeon Jin Lee is a computer scientist in the Intelligent Robotics Group at NASA. She is a software developer for Exploration Ground Data Systems (xGDS), through which she supported projects such as Pavilion Lake Research Project, Mojave Volatile Field Prospector, and BASALT. She is also a software lead for Geocam Space Project, a tool for semi-automatic and automatic registration of Earthobserving images taken from the ISS. She received her B.S. in Electrical Engineering and Computer Science from UC Berkeley, and M.S. in Software Engineering from Carnegie Mellon University.

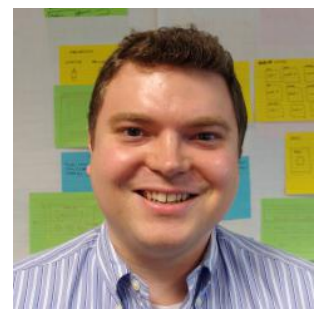

Steven Hillenius leads the Playbook team in the Human Systems Integration Division at NASA Ames Research Center. He has designed mission planning software used by astronauts, scientists, and engineers for the International Space Station, Mars Rover Curiosity, LADEE (Lunar Atmosphere Dust Environment Explorer), and several of the NASA Analog
Missions. Recently, his work involves designing and building software for astronauts to plan and run their own missions in deep space where connectivity back to Earth is limited and researching usable technology to make training and space operations more efficient. Steven received a Master's in Human-Computer Interaction and B.S. in Information Systems and Human-Computer Interaction, both from Carnegie Mellon University.

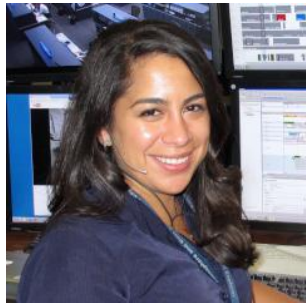

Ivonne Deliz is a software developer in the Playbook team at NASA Ames Research Center. She has a Bachelor's degree in Computer Science from the University of Puerto Rico, Rio Piedras, and a Master's in Computer Science (Vision and Graphics) from Columbia University. She developed SPIFe planning and scheduling tools for various International Space Station Mission Control consoles, Mars Science Laboratory (Curiosity) and LADEE Lunar Orbiter. She is the Lead Planner for the NEEMO Analog Mission and has trained aquanauts on Playbook use for mission operations. She also supports the BASALT Analog Mission for on-site technical and timelining support.

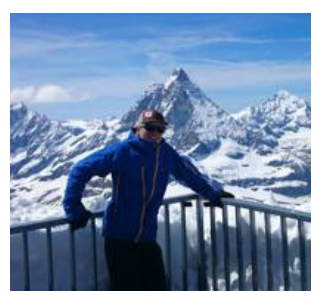

Johannes Norheim is a graduate research assistant in the Man Vehicle Lab at MIT's Department of Aeronautics and Astronautics, advised by Dr. Jeffrey Hoffman. He received a Bachelor's degree in Aerospace Engineering(2016) from the same institute. He has experience in space systems design and engineering area through work at the Dutch National Aerospace Laboratory, Thales Alenia Space and several university competitions ranging from CubeSat to space station design. He also has experience in the domain of path and activity planning through undergraduate research in the Model-based Embedded Robotic Systems(MERS) Lab at MIT Computer Science and Artificial Intelligence Laboratory(CSAIL), where he worked on visualization tools for plans and a closed loop planning execution framework for robots.

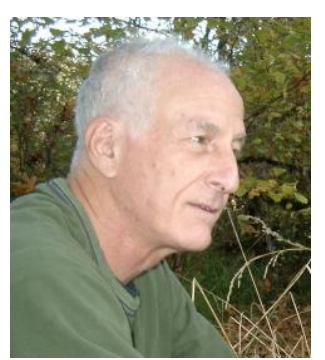

Jeffrey Hoffman is a professor in MIT's Aeronautics and Astronautics Department. He received a $B A$ in Astronomy (summa cum laude) from Amherst College (1966); a PhD in Astrophysics from Harvard University (1971); and an MSc in Materials Science from Rice University (1988). As a NASA astronaut (1978-1997) Dr. Hoffman made five space flights, becoming the first astronaut to log 1000 hours of flight time aboard the Space Shuttle. He was a member of 
the spacewalking team that repaired the optics of the Hubble Space Telescope. His primary research interests are in improving the technology of space suits and designing innovative space systems for human and robotic space exploration. Dr. Hoffman is director of the Massachusetts Space Grant Consortium. In 2007, Dr. Hoffman was elected to the US Astronaut Hall of Fame.

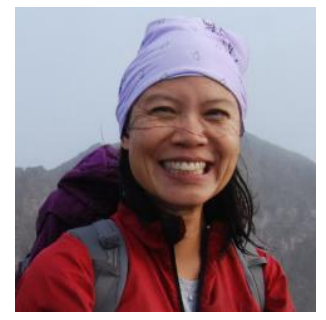

Darlene Lim received her Ph.D. from the University of Toronto during which she conducted climate change research in the Canadian High Arctic. She is now based at the NASA Ames Research Center, and her work as a geobiologist has since extended to include the development of operations concepts and capabilities in support of human-robotic scientific exploration of our Solar System. Darlene has spent over two decades leading field research around the world and is the Principal Investigator (PI) of BASALT and PLRP. 